\title{
Clip-off Chemistry: Synthesis by Programmed Disassembly of Reticular Materials
}

Yunhui Yang ${ }^{1, \dagger}$, Anna Broto-Ribas ${ }^{1, \dagger}$, Borja Ortín-Rubio ${ }^{1, \dagger}$, Inhar Imaz ${ }^{1, *}$, Felipe Gándara ${ }^{2}$, Arnau Carné-Sánchez ${ }^{1}$, Vincent Guillerm ${ }^{1}$, Sergio Jurado ${ }^{3}$, Félix Busqué ${ }^{3}$, Judith Juanhuix ${ }^{4}$, Daniel Maspoch ${ }^{1,5, *}$

\author{
Affiliations: \\ ${ }^{1}$ Catalan Institute of Nanoscience and Nanotechnology (ICN2), CSIC and The Barcelona \\ Institute of Science and Technology, Campus UAB, Bellaterra, 08193 Barcelona, Spain. \\ ${ }^{2}$ Department of New Architectures in Materials Chemistry, Materials Science Institute of \\ Madrid - CSIC, Sor Juana Inés de la Cruz 3, Madrid 28049, Spain. \\ ${ }^{3}$ Departament de Química, Universitat Autònoma de Barcelona (UAB), Cerdanyola del \\ Vallès, 08193, Spain. \\ ${ }^{4}$ ALBA Synchrotron, 08290 Cerdanyola del Vallès, Barcelona, Spain. \\ ${ }^{5}$ ICREA, Pg. Lluís Companys 23, 08010 Barcelona, Spain.
}

*Corresponding authors. Email: inhar.imaz@icn2.cat and daniel.maspoch@icn2.cat $\dagger$ These authors contributed equally to this work.

\begin{abstract}
Bond-breaking is an essential process in natural and synthetic chemical transformations. Accordingly, the ability for researchers to strategically dictate which bonds in a given system are broken translates to greater synthetic control, as historically evidenced in fields such as organic synthesis. Here, we report extending the concept of selective bondbreaking to reticular materials, in a new synthetic approach that we call Clip-off Chemistry. We show that bond-breaking in these structures can be controlled at the molecular level; is periodic, quantitative and selective; is effective in reactions performed in either solid or liquid phases; and can occur in a single-crystal-to-single crystal fashion involving the entire bulk precursor sample. Clip-off Chemistry opens the door to programmed disassembly of reticular materials and thus, to the design and synthesis of new molecules and materials.
\end{abstract}

One Sentence Summary: Bond-breaking in reticular materials enables their programmed disassembly for synthesis of new molecules and materials. 


\section{Main Text:}

Throughout history, innovations in chemical synthesis have yielded previously inaccessible new molecules and materials that have enabled vast improvements in human life, ranging from fine chemicals to complex functional materials. Every new reaction and methodology not only help to expand accessible chemical space, but also inspire researchers to further innovate in the iterative design and preparation of new chemical targets of social, economic or industrial value. To date, most state-of-the-art synthetic approaches use bottomup strategies that, at the latter stage, mainly entail controlling the formation of new bonds. A relatively recent example of this is reticular chemistry (1-7), in which judiciously designed, rigid, molecular building blocks (MBBs) are linked by strong bonds to create crystalline openframework materials (8), such as metal-organic frameworks (MOFs) (1-4), covalent-organic frameworks (COFs) (9) and metal-organic polyhedra (MOPs) (10-12).

Reticular materials are a fascinating source of metal-organic and purely organic structures built up from an endless variety of fragments and MBBs (13) (e.g. metal clusters, cages, cycles, metal layers, metal chains, etc.) that often do not exist in their isolated form. We envisaged that by selectively breaking certain bonds in such reticular materials, we could transform them into new frameworks or to break them into new molecular fragments or isolated MBBs, as a synthetic strategy to new materials and molecules. We hypothesized that we could use a chemical reaction for programmed bond-breaking, so that the de-reticulation process would occur at the molecular level. We reasoned that this would require the presence of cleavable groups at specific positions within the structures of the corresponding reticular materials. Reported reticular materials that feature linkers containing alkene bonds would be ideal starting materials for this strategy. However, we reasoned that, in the likely event that the targeted structure-precursor does not contain any alkene groups, then we could generalize such an approach to numerous reticular materials by inserting such groups into the pre-selected linkers without modifying their size or geometry, via reticular chemistry. Indeed, reticular chemistry dictates that for a given framework, the constituents can be chemically functionalized pre- and/or post-synthetically $(14,15)$, without any loss to framework connectivity. This idea translates to the ability to encode the organic linker of an existing MOF by inserting cleavable alkene groups, without modifying the linker size or topology, to enable assembly of the corresponding isoreticular MOF structures containing the desired cleavable group (16).

Here we report a new synthetic approach, called Clip-off Chemistry, which is based on the programmed disassembly of reticular materials by controlling the breaking of bonds. We validated Clip-Off Chemistry by synthesizing two topologically distinct, three-dimensional (3D) MOFs from two reported 3-D MOFs in single-crystal-to-single-crystal transformations. Through these examples, we demonstrated that reticular materials could be modified via cleavage, rather than formation, of bonds in their frameworks, enabling alterations to the connectivity of their constituent MBBs and therefore, to their topology. Next, we anticipated that Clip-off Chemistry could be generalized by applying it to 0-D molecular systems. To this end, we synthesized a novel metal-organic macrocycle from a MOP precursor. In all the examples we report here, we used ozonolysis as the chemical reaction to cut off constituent organic MBBs or linkers via direct cleavage of their alkene bonds.

\section{Reaction design in clip-off chemistry: synthesis of a first 3-D MOF}

Clip-off chemistry is based on using, as starting materials, structures that contain cleavable groups (in this study, alkenes) at specific positions, such that cleavage of said groups generates 
new molecules or materials. Following this principle, we first targeted the clip-off synthesis of a new 3-D MOF from a 3-D MOF precursor. When selecting precursors in clip-off chemistry, reticular analysis of their underlying nets (17-19) is important as bond-breaking in the structure relates to disassembly of certain circuits of connections (20). In reticular materials, this disassembly can be performed mainly by erasing some of the edges or nodes. Translating this analysis to the chemical field, the clip-off synthesis of a structure (in this example, a 3-D MOF) from the disassembly of another structure (in this example, another 3-D MOF) can be achieved by selecting either of two potential precursors i) a structure built from one type of polytopic linker that contains different circuits of connections between the clusters, containing in at least one of the circuits a cleavable bond; or ii) a mixed-linker structure, where linkers are localized at different crystallographic positions and one of them contains a cleavable bond. Cleavage of the polytopic linker will provoke disassembly of the circuits of connections in which the cleavable bond is involved, thereby forming a structure built up exclusively from those circuits that do not contain the cleavable groups, with a distinct underlying topology. Similarly, in the mixed-linker structure, cleavage of the linker containing the cleavable bond will provoke disassembly of the circuits of connections in which it is involved, thereby forming a structure built up exclusively from those circuits that involve the metal clusters and the other linkers that do not contain the cleavable groups.

In our choice of precursor for the first 3-D MOF synthesis, we followed the polytopic linker approach described above (Fig. 1). Thus, we selected the Zr-scu-MOF (21), in which the assembly of eight-connected (8-c), quadrangular prismatic $\mathrm{Zr}_{6} \mathrm{O}_{4}(\mathrm{OH})_{4}$ clusters to 4-c rectangular linkers 5-[(3,5-dicarboxyphenyl)diazenyl]benzene-1,3-dicarboxylate $\left(\mathrm{L}_{1}\right)$ forms a 3-D framework with a 4,8-c scu/3,3,8T132 underlying topology (22) and 1-D channels (size: $\sim 7 \AA$ ) along one direction. However, as this reported structure does not contain cleavable alkene groups, we had to insert them chemically, using the well-known isoreticular principle (16). This step of introducing cleavable groups into precursors can be performed before the cleavage step as needed, by replacing at least one of the original linkers with one of similar size and geometry that contains the desired cleavable groups at strategic positions. Once inserted into the isoreticular structure, such linkers generate different circuits of connections, in which at least one circuit contains the cleavable bonds. In our example, we replaced the linker $\mathrm{L}_{1}$ with 5-[2-(3,5-dicarboxyphenyl)ethenyl]benzene-1,3-dicarboxylate ( $\left.\mathrm{L}_{2}\right)$, which contains olefinic bonds for cleavage. We thus synthesized an isoreticular $\mathrm{Zr}$-scu-MOF framework built up from different circuits of connections, wherein only some of them contain the cleavable olefinic bonds of $\mathrm{L}_{2}$. We anticipated that, upon treatment of this framework with ozone (23-27), each 4-c L linker would be split into two 2-c linkers (doubly deprotonated trimesic acid and/or 5-formylisophthalate). Consequently, only the circuits of connections between the $\mathrm{Zr}_{6} \mathrm{O}_{4}(\mathrm{OH})_{4}$ clusters and these 2-c linkers would remain, forming a $\mathrm{Zr-pcu}-\mathrm{MOF}$. In contrast to the isoreticular $\mathrm{Zr}$-scu-MOF precursor, which is based on quadrangular prismatic 8-c $\mathrm{Zr}$-clusters, each $\mathrm{Zr}_{6} \mathrm{O}_{4}(\mathrm{OH})_{4}$ cluster is still coordinated by 8 linkers in this new $\mathrm{Zr-pcu}$ MOF, however its topological connectivity decreases from 8-c to a 6-c because two pairs of the eight 2-c linkers connect the same two clusters (fig. S2) (28). 


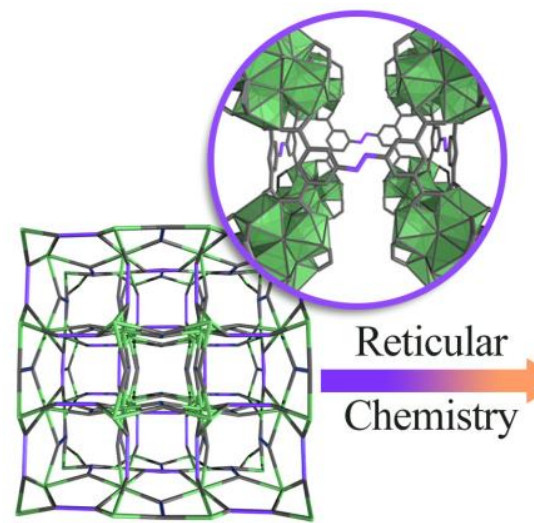

Zr-scu-MOF

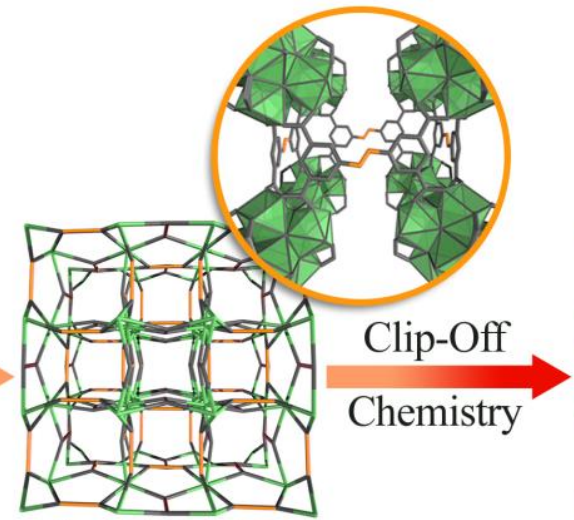

isoreticular Zr-scu-MOF

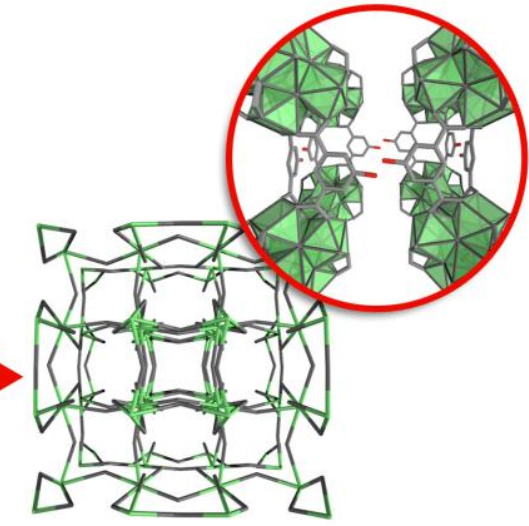

Zr-pcu-MOF

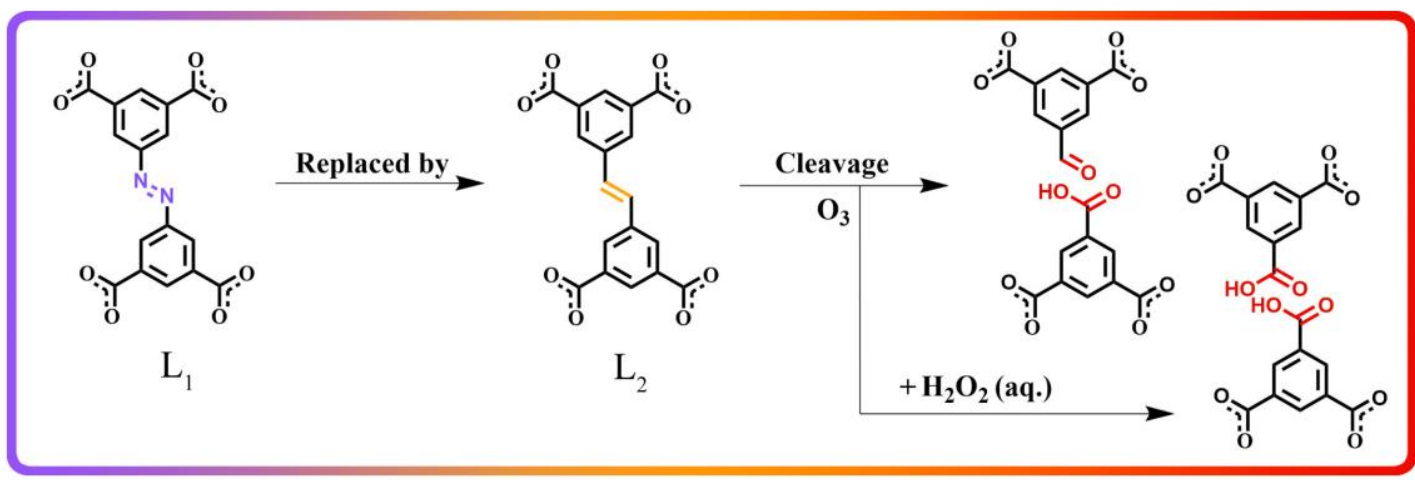

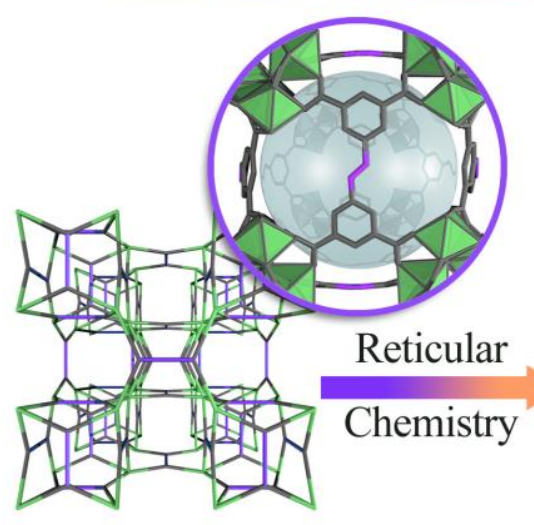

Sc-soc-MOF

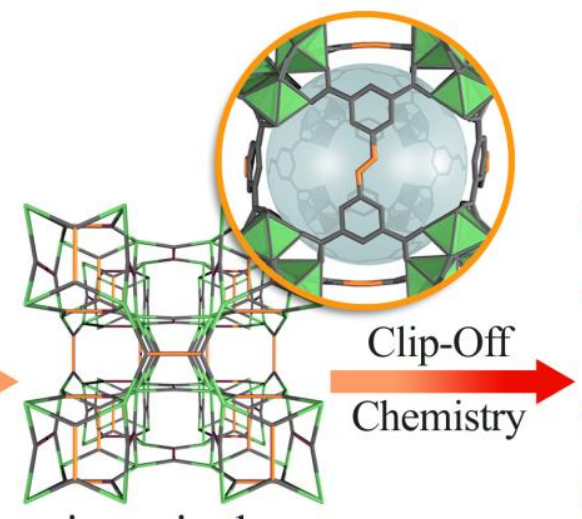

isoreticular

Sc-soc-MOF

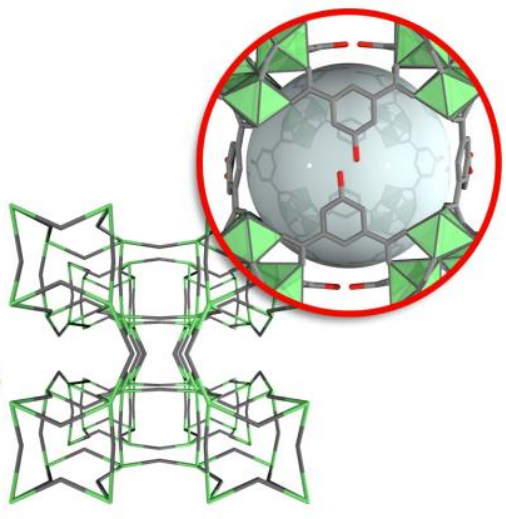

Sc-pcu-MOF

Fig 1. Clip-off chemistry approach. Shown is the synthetic steps that define the clip-off chemistry, starting with the insertion of cleavable (alkene) groups in a MOF structure through the isoreticular approach (16) and followed then by the quantitative break of these cleavable (alkene) groups through ozonolysis to synthesize a new structure. Top and bottom parts show these steps for the clip-off synthesis of Zr-pcu-MOF and Sc-pcu-MOF from Zr-scu-MOF and Sc-soc-MOF, respectively. Middle part shows the chemistry involved in this approach. The formation of the aldehyde/carboxylic acid groups after ozonolysis are not shown in the lower magnification nets for better illustration of the breaking process. The formation of these groups is shown in the magnified structures, wherein the red groups represent aldehyde or carboxylic acid groups. 
We expected that this first clip-off reaction would afford the first-ever example of a 3D MOF made of archetypical Zr-oxo-hydroxo-clusters linked by isophthalate-like linkers in a pcu underlying net. Interestingly, the closest literature example of such a structure is that of a 2-D MOF made of Zr-oxo-hydroxo-clusters linked by isophthalate but in an hcb topology (29). Our target 3-D Zr-pcu MOF could also be seen as exemplifying a connection of Zr-oxohydroxo-clusters through trimesate linkers, which are coordinated only through two of their three carboxylate groups. Accordingly, it could also be considered as the first example of a $\mathrm{Zr}$ structure with a free carboxylic acid using trimesate, as researchers have previously reported combination of Zr-oxo-hydroxo-clusters with this linker in a spn topology of 3,6-connectivity $(30)$.

\section{Clip-off synthesis: from Zr-scu-MOF to Zr-pcu-MOF}

We synthesized colorless cubic crystals of isoreticular Zr-scu-MOF by solvothermal reaction $\left(120{ }^{\circ} \mathrm{C}\right)$ of a mixture of $\mathrm{ZrOCl}_{2} \cdot 8 \mathrm{H}_{2} \mathrm{O}$ and $\mathrm{L}_{2}$ in $N, N$-dimethylformamide (DMF) and formic acid for 5 days. The crystal structure revealed the expected isoreticular scu framework, wherein the $\mathrm{L}_{1}$ had been replaced by $\mathrm{L}_{2}$ (Fig. 2A and section S2). Next, we confirmed the phase purity of the bulk sample by X-ray powder diffraction (XRPD) and scanning electron microscopy (SEM) (Fig. 2B and S3,12).

We began the clip-off synthesis of $\mathrm{Zr}$-pcu-MOF by packing $20 \mathrm{mg}$ of synthesized $\mathrm{Zr}$ scu-MOF crystals into a plastic tube, connected on one side to the ozonator (through a $\mathrm{CaCl}_{2}$ humidity trap), and on the other side, to a vacuum pump (through a KI trap), to ensure a continuous flow of ozone through the column $(23,24)$. The reaction was run at room temperature for $30 \mathrm{~min}$. Afterwards, the sample was connected to vacuum for another $30 \mathrm{~min}$. This ozonation/vacuum cycle was repeated nine times for the complete cleavage of all the alkene bonds in $\mathrm{Zr}$-scu-MOF, as evidenced by the gradual disappearance of the characteristic olefinic peak at $7.69 \mathrm{ppm}$ in the ${ }^{1} \mathrm{H}$ NMR spectra of the digested $\mathrm{Zr}$-scu-MOF crystals (vide infra; fig. S8). After nine cycles, the resulting solid was directly collected from the tube and stored in tetrahydrofurane.

We then characterized the ozonated $\mathrm{Zr}$-scu-MOF crystals by single crystal X-ray diffraction (SCXRD), which confirmed that they had retained the single-crystal character (Fig. $2 \mathrm{C}$ and $\mathrm{S} 1,2)$. For these crystals, synchrotron diffraction data were collected at a maximum resolution of $1.2 \AA$. Data analyses revealed a variation in the lattice parameters, as compared to the pristine structure. Both pre- and post-ozonolysis crystal structures were solved in the monoclinic $C 2 / \mathrm{m}$ space group. However, the cell volume of the ozonated crystal was found to be $16076.00(10) \AA^{3}$, whereas that of the pristine $\mathrm{Zr}$-scu-MOF was $16957.46(10) \AA^{3}$. Analysis of the diffraction data demonstrated the integrity of the inorganic $\mathrm{Zr}$-oxo-hydroxo-clusters after post-synthetic reaction, with no changes in their coordination. Thus, the position of all the atoms in the inorganic secondary building units (SBUs, metal and carboxylate atoms) were assigned and anisotropically refined. As for the rest of the organic linker, carbon atoms belonging to the phenyl ring were located in the difference electron density maps. However, their high thermal parameter values indicated a possible positional disorder. Moreover, the olefinic carbon atoms could no longer be located, supporting the completeness of the ozonolysis reaction. This is in stark contrast to the pristine MOF, where the positions of these atoms were clearly visible and refined. The position of the resulting aldehydes or carboxylic groups, newly inserted in the MOF upon cleavage of the carbon-carbon double bond, could not be determined from the analysis of the difference electron density maps. Instead, a large residual electron density was observed in the vicinity of the positions that were initially occupied by the olefinic group. Additional differences could be observed in the disposition of 
other framework atoms when comparing both structures. Most notably, the relative orientation of opposite phenyl rings that were part of a same linker in $\mathrm{Zr}$-scu-MOF is different after ozonolysis (Fig. 2A,C), indicating that the olefin bond is no longer present to fix the angle between these rings.

As pristine Zr-scu-MOF and ozonated Zr-scu-MOF (or Zr-pcu-MOF) showed marked differences in their crystal structures, we also studied them by XRPD. Remarkably, the XRPD patterns for each sample perfectly matched those calculated from the corresponding structures determined by SCXRD and full pattern profile refinements further demonstrated phase purity of the samples (Fig. 2B and S3-5). Altogether, these results corroborate two crucial phenomena for validation of Clip-off Chemistry: firstly, that it is indeed possible to cleave all the periodic olefinic bonds in a 3D structure without destroying it or its single-crystal character; and secondly, that said cleavage and the resultant formation of a new structure (in this case, $\mathrm{Zr}$ pcu-MOF (19)) are homogeneous throughout the bulk crystalline sample.

Next, we gained further evidence of the formation of Zr-pcu-MOF by measuring the ${ }^{1} \mathrm{H}$ NMR spectra of the digested ozonized samples (5\% HF/DMSO- $d_{6}$ ) after each cycle, and then comparing each spectrum to that of the starting Zr-scu-MOF (Fig. 2D and S6-8). Under these digestion conditions, the MOF structures are destroyed and the linkers released, which makes possible using ${ }^{1} \mathrm{H}$ NMR to unveil the composition of the initial MOF structures. The spectrum of the digested $\mathrm{Zr}$-scu-MOF showed the characteristic peak of equivalent olefinic protons of $\mathrm{L}_{2}$ at $\delta=7.69 \mathrm{ppm}$. In contrast, the spectra of ozonated crystals after each cycle confirmed a gradual fading of this olefinic signal, which, by the ninth cycle, had fully disappeared. Indeed, ${ }^{1} \mathrm{H}$ NMR of digested ozonated Zr-scu-MOF after the ninth cycle confirmed the full conversion of $\mathrm{L}_{2}$ into doubly deprotonated trimesic acid and 5formylisophthalate. Beyond the disappearance of the olefinic protons at $\delta=7.69 \mathrm{ppm}$, there was also fading of the phenyl $(\delta=8.49 \mathrm{ppm}$ and $8.39 \mathrm{ppm})$ and the carboxylic acid $(\delta=13.32$ ppm) protons of $\mathrm{L}_{2}$, while the characteristic signals for the trimesic acid $(\delta=13.57 \mathrm{ppm}$ and $8.65 \mathrm{ppm})$ and 5-formylisophthalic acid $(\delta=10.18 \mathrm{ppm}, 8.70 \mathrm{ppm}$ and $8.64 \mathrm{ppm})$ were clearly identifiable. Cleavage of the olefinic bonds was corroborated by negative-mode mass spectrometry and Fourier transform infrared (FT-IR) spectroscopy of both pristine and ozonated $\mathrm{Zr}$-MOFs (figs. S10,11). In the mass spectrum, the molecular ions $[\mathrm{M}-\mathrm{H}]^{-}$ corresponding to trimesic acid $(\mathrm{m} / \mathrm{z}=209.01)$ and 5 -formylisophthalic acid $(\mathrm{m} / \mathrm{z}=193.01)$ were found, in strong contrast to the absence of any molecular ion $[\mathrm{M}-\mathrm{H}]^{-}$corresponding to $\mathrm{L}_{2}$ $(\mathrm{m} / \mathrm{z}=355.05)$. Similarly, in comparison to the spectrum of pristine $\mathrm{Zr}$-scu-MOF, the FT-IR spectrum of ozonated $\mathrm{Zr}$-pcu-MOF revealed the appearance of a typical carbonyl $(\mathrm{C}=\mathrm{O})$ stretching band at $1700 \mathrm{~cm}^{-1}$ and the complete disappearance of an alkene $(\mathrm{C}=\mathrm{C})$ stretching band at $1622 \mathrm{~cm}^{-1}$.

Having demonstrated the single-crystal-to-single-crystal synthesis of Zr-pcu-MOF by selectively cleaving the olefinic bonds in $\mathrm{Zr}$-scu-MOF into a mixture of aldehyde and carboxylic acid groups, we next sought to perform analogous chemistry to synthesize Zr-pcuMOF functionalized exclusively with carboxylic acids; thus, showing improved control on the final output of the clip-off chemistry. To this end, ozonated crystals were soaked in $1 \mathrm{~mL}$ of hydrogen peroxide ( $30 \mathrm{wt}$ \% in $\mathrm{H}_{2} \mathrm{O}$ ) solution for three weeks. During this period, the sample was refreshed with fresh hydrogen peroxide every $24 \mathrm{~h}$. Next, the sample was centrifuged and washed twice with water. The transformation to carboxylic acid groups was quantitative, as confirmed by ${ }^{1} \mathrm{H}$ NMR analysis (Fig. 2D and S9). Moreover, the Zr-pcu-MOF framework had been preserved, as confirmed by XRPD (Fig. 2B and S3). 
A

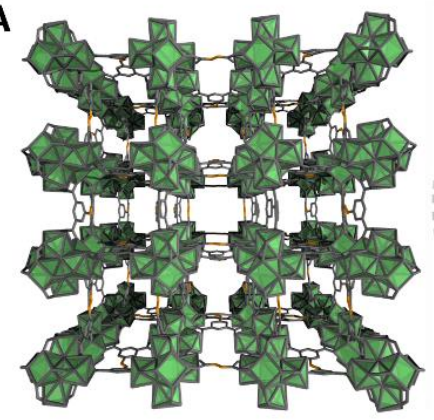

C

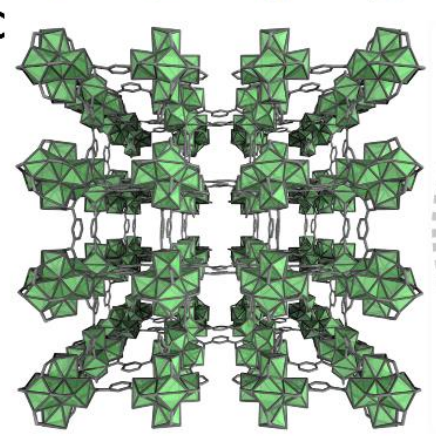

E

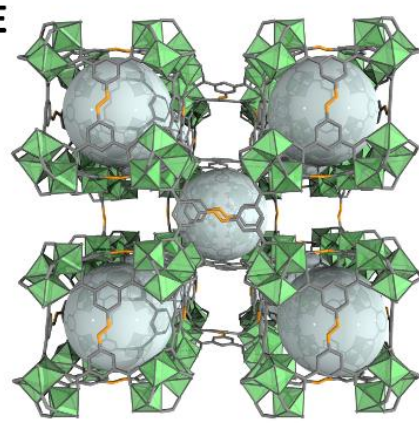

G

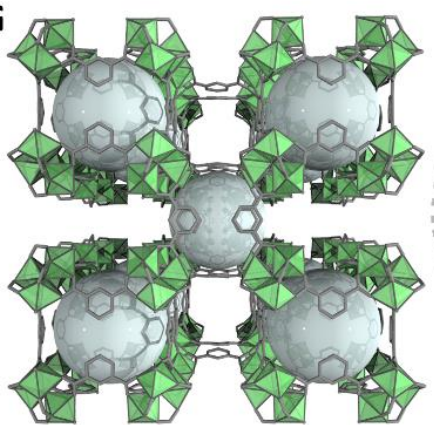

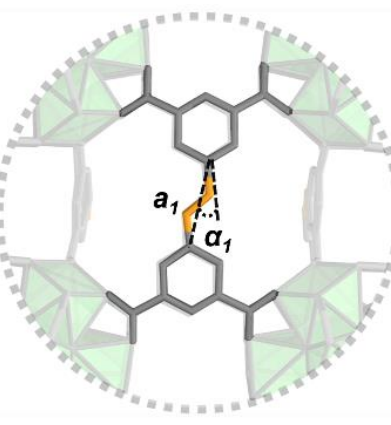
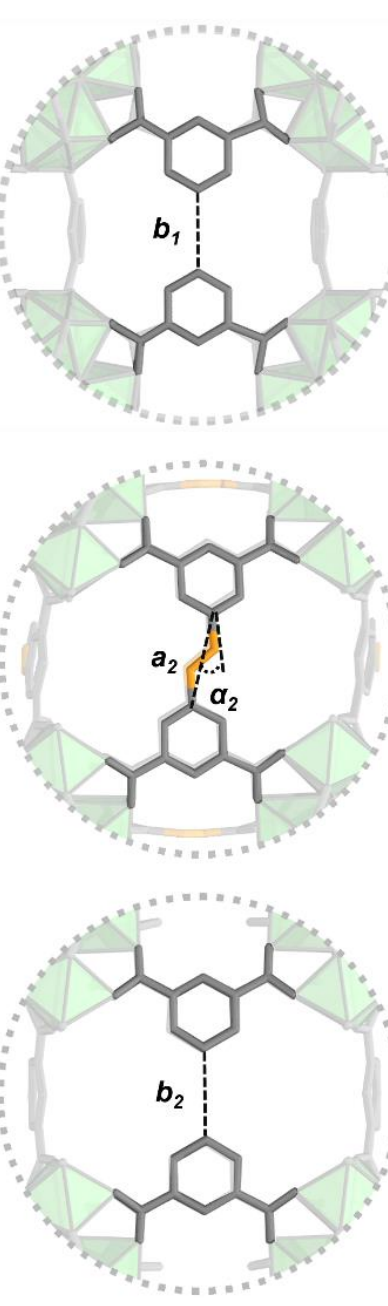

B

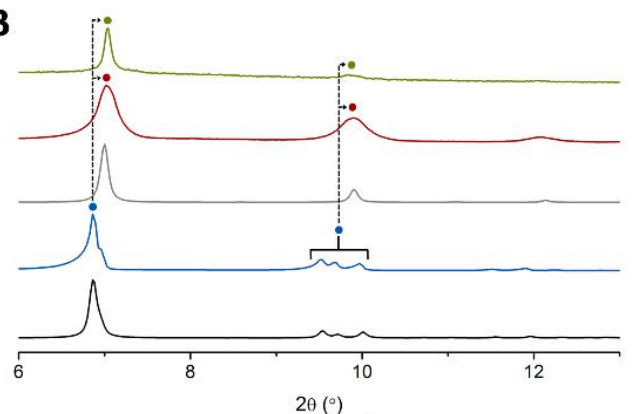

D

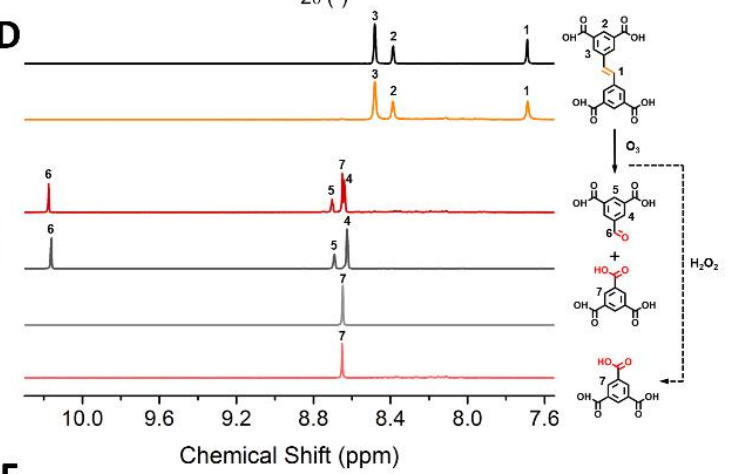

$\mathbf{F}$

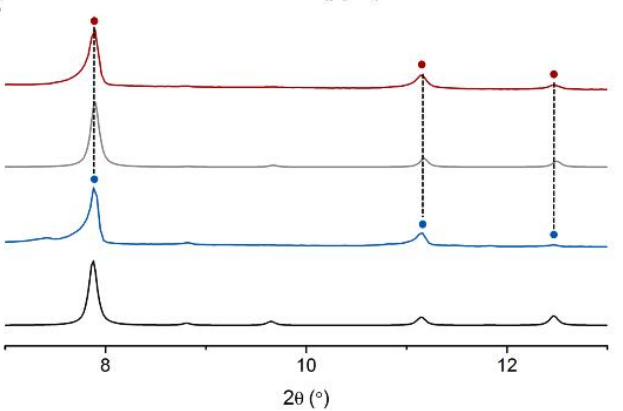

H

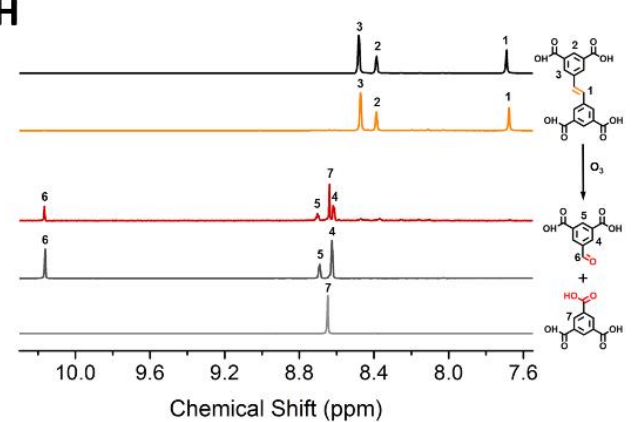

Fig 2. Structural and molecular characterization of $\mathrm{Zr}$-pcu-MOF and Sc-pcu-MOF. $(\mathbf{A}, \mathbf{C})$ Crystal structures of isoreticular Zr-scu-MOF (A) and Zr-pcu-MOF (C). Magnified views highlight the changes observed in the distances and angles of both phenyl rings (from $a_{1}$ $=3.77-3.79 \AA$ and $\alpha_{1}=14.95-19.71^{\circ}$ to $b_{1}=3.39-3.65 \AA$ and $\beta_{1}=0^{\circ}$ ) initially composing $\mathrm{L}_{2}$. (B) Magnified view of XRPD of calculated Zr-scu-MOF (black), synthesized Zr-scu-MOF (blue), calculated Zr-pcu-MOF (grey), synthesized Zr-pcu-MOF (red) and oxidized Zr-pcuMOF (green). Main structural changes with signal shifts are highlighted. (D) ${ }^{1} \mathrm{H}$ NMR spectra (360 MHz, DMSO- $d_{6}$ ) of digested $\mathrm{L}_{2}$ (black), Zr-scu-MOF (orange), Zr-pcu-MOF (red), 5formylisophthalic acid (dark grey), trimesic acid (light grey) and oxidized Zr-pcu-MOF (light red). Assigned proton signals: $\delta_{1}=7.69(\mathrm{~s}, 2 \mathrm{H}), \delta_{2}=8.39(\mathrm{~s}, 2 \mathrm{H}), \delta_{3}=8.49(\mathrm{~s}, 4 \mathrm{H}), \delta_{4}=8.64$ $(\mathrm{m}, 2 \mathrm{H}), \delta_{5}=8.70(\mathrm{~m}, 1 \mathrm{H}), \delta_{6}=10.18(\mathrm{~s}, 1 \mathrm{H})$ and $\delta_{7}=8.65(\mathrm{~s}, 3 \mathrm{H})$. (E,G) Crystal structures 
of isoreticular Sc-soc-MOF (E) and Sc-pcu-MOF (G). Magnified views highlight the changes observed in the distances and angles of both phenyl rings (from $a_{2}=3.82-3.84 \AA$ and $\alpha_{2}=$ $15.63-17.34^{\circ}$ to $a_{2}=3.62-3.63 \AA$ and $\beta_{2}=0^{\circ}$ ) initially composing $L_{2}$. (F) Magnified view of XRPD of calculated Sc-soc-MOF (black), synthesized Sc-soc-MOF (blue), calculated Sc-pcuMOF (grey) and synthesized Zr-pcu-MOF (red). (H) ${ }^{1} \mathrm{H}$ NMR spectra (360 MHz, DMSO-d6) of digested $\mathrm{L}_{2}$ (black), Sc-soc-MOF (orange), Sc-pcu-MOF (red), 5-formylisophthalic acid (dark grey) and trimesic acid (light grey). Assigned proton signals: $\delta_{1}=7.68(\mathrm{~s}, 2 \mathrm{H}), \delta_{2}=8.38$ $(\mathrm{s}, 2 \mathrm{H}), \delta_{3}=8.47(\mathrm{~s}, 4 \mathrm{H}), \delta_{4}=8.64(\mathrm{~m}, 2 \mathrm{H}), \delta_{5}=8.71(\mathrm{~m}, 1 \mathrm{H}), \delta_{6}=10.17(\mathrm{~s}, 1 \mathrm{H})$ and $\delta_{7}=8.65$ $(\mathrm{s}, 3 \mathrm{H})$.

\section{Clip-off synthesis: from Sc-soc-MOF to Sc-pcu-MOF}

To demonstrate that Clip-off Chemistry could be generalized to other 3D MOF structures, we next attempted to synthesize a 3-D Sc-pcu-MOF from an Sc-soc-MOF precursor (31) (Fig. 1 and section S3), a 3-D structure built up from linking 6-c trigonal, prismatic $\mathrm{Sc}_{3} \mathrm{O}$ clusters to 4-c $\mathrm{L}_{1}$ linkers in a 4,6-c soc/edq topology (32). This structure exhibits a mixture of cubic cavities (size: $11 \AA$ ) and 1-D channels (size: $8 \AA$ ) along the three axes. As in the previous case, we designed the precursor by replacing $\mathrm{L}_{1}$ with $\mathrm{L}_{2}$, thus forming an isoreticular Sc-socMOF that contains different circuits of connections, some of which contains the cleavable olefins. We envisioned that, upon treatment with ozone, this circuit would be disrupted to form a 3-D Sc-pcu-MOF whose $\mathrm{Sc}_{3} \mathrm{O}$ clusters would be connected through trimesate/5formylisophthalate linkers.

We began the clip-off synthesis of Sc-pcu-MOF via the aforementioned route, by first preparing the isoreticular Sc-soc-MOF precursor. Colorless cubic crystals of Sc-soc-MOF were prepared by heating a solution of $\mathrm{Sc}\left(\mathrm{NO}_{3}\right)_{3} \cdot \mathrm{xH}_{2} \mathrm{O}$ and $\mathrm{L}_{2}$ in $\mathrm{DMF}$, ethanol and formic acid at $120{ }^{\circ} \mathrm{C}$ for $48 \mathrm{~h}$ (Fig. 2E). However, all attempts at complete ozonolysis were unsuccessful, whether using the same synthetic protocol as for Zr-scu-MOF or trying slight modifications (e.g. extending the reaction time up to $8 \mathrm{~h}$ and/or the number of ozonolysis cycles up to 12; figs. S13-16). Consequently, cleavage of the olefinic bonds was incomplete, reaching a maximum value of $60 \%$ to $70 \%$ under certain conditions (e.g. exposing the Sc-soc-MOF crystals to ozone gas for $2 \mathrm{~h}$ ). Consequently, a distinct ozonolysis protocol was employed: Scsoc MOF crystals were immersed in water, and then ozone was bubbled through the suspension under stirring at room temperature for $12 \mathrm{~h}$. These conditions afforded greater cleavage of the olefinic bonds than in the previous strategy, leading to a mixture of trimesic acid, 5formylisophthalic acid and the intermediate 1,2,4-trioxolane ring (fig. S17). Importantly, presence of this intermediate indicated that the olefinic bonds had not all been completely cleaved to aldehyde/carboxylic acid groups. Moreover, increasing the reaction time under these solid/liquid conditions did not provide any major improvement in cleavage, although it did lead to a slight decrease in the crystallinity of the ozonated Sc-pcu-MOF.

To address the aforementioned drawbacks, we decided to combine the two ozonolysis strategies, starting with a solid-gas reaction for $2 \mathrm{~h}$ and then, cleaving the remaining alkene bonds via suspension/gas reaction for an additional $6 \mathrm{~h}$. Under these conditions, SCXRD of the resulting crystals confirmed quantitative single-crystal-to-single-crystal cleavage of the olefinic bonds of Sc-soc-MOF to synthesize Sc-pcu-MOF (19) (Fig. 2G). However, in this case, no changes were observed in the volume of the $R-3$ rhombohedral unit cell relative to that of the precursor, as also confirmed by XRPD (Fig. 2F and S20). Nevertheless, SCXRD analysis showed a very similar case to that of ozonated Zr-scu-MOF. Thus, the connectivity of the atoms belonging to the inorganic trimeric $\mathrm{ScO}_{3}$ clusters remained identical to that of the pristine $\mathrm{Sc}-$ 
soc-MOF. Refinement of the carbon atoms from the organic linkers evidenced a positional disorder for some of them, and, unlike Sc-soc-MOF, the Sc-pcu-MOF product did not contain any olefinic atoms. Instead, areas of electron density were observed only between the opposite phenyl rings, with a maximum value at the middle point between the two rings, but at a distance longer than that expected for a $\mathrm{C}=\mathrm{C}$ bond. This area of higher electron-density points perpendicularly above and below the plane of the phenyl rings. Although the refinement indicates the presence of atoms in this area, these atoms could not be unambiguously assigned to aldehyde or carboxylate groups (fig. S18). These findings indicate that the functional groups are not arranged in an orderly fashion within the crystals after ozonolysis, but are rather randomly oriented in the pores. This is not surprising, considering that the resulting aldehydes and/or carboxylic groups are random distributed and most likely dangle in different orientations into the pores, rather than having a periodically fixed position; accordingly, they would not contribute to the periodic diffraction of the crystal. Consistent with these SCXRD data, cleavage of all olefinic bonds was confirmed by ${ }^{1} \mathrm{H}$ NMR (Fig. $2 \mathrm{H}$ and S21-23) and negativemode mass spectrometry of the digested ozonated samples (5\% HF/DMSO- $d_{6}$ ) (fig. S24), and FT-IR of ozonated Sc-pcu-MOF (fig. S25), from which the olefinic protons $(\delta=7.68 \mathrm{ppm})$, molecular ion $[\mathrm{M}-\mathrm{H}]^{-}$of $\mathrm{L}_{2}$ and the typical alkene $(\mathrm{C}=\mathrm{C})$ stretching band, respectively, were not observed. Contrariwise, the ${ }^{1} \mathrm{H}$ NMR signals, the molecular ions $\left.[\mathrm{M}-\mathrm{H}]\right]^{-}$and the carbonyl $(\mathrm{C}=\mathrm{O})$ stretching band corresponding to doubly deprotonated trimesic acid and 5formylisophthalate were all clearly identifiable, thus further corroborating the clip-off synthesis of Sc-pcu-MOF.

\section{Extending clip-off chemistry to a 0-D system: from a Rh-MOP to a Rh-macrocycle}

Finally, we endeavored to use Clip-off Chemistry to synthesize a metal-organic macrocycle (33-36) from a 0-D MOP system. To this end, we followed the second aforementioned approach, designing a mixed-linker Rh-MOP that we could transform into a triangular metal-organic macrocycle (Fig. 3A and section S4). We selected a Rh-MOP $(37,38)$ built from six 4-c $\mathrm{Rh}$ paddle-wheel clusters linked by six 5-tert-butylbenzene-1,3dicarboxylate $\left(\mathrm{L}_{3}\right)$ linkers and by six 3-[(3-carboxyphenyl)diazenyl]benzoate $\left(\mathrm{L}_{4}\right)$ linkers, forming a cage with a diameter of $12 \AA$. In this cage, three paddle-wheel clusters are linked by three $\mathrm{L}_{3}$ linkers to form a triangular macrocycle, and two of these units are linked by six $\mathrm{L}_{4}$ linkers. Following a similar strategy as those explained in the previous examples, we functionalized this MOP with alkene groups by replacing $\mathrm{L}_{4}$ with 3-[2-(3carboxyphenyl)ethenyl]benzoate ( $\left.\mathrm{L}_{5}\right)$. Accordingly, the isoreticular Rh-MOP would contain a non-cleavable $\mathrm{L}_{3}$ linker and a cleavable $\mathrm{L}_{5}$ linker, which, upon cleavage via ozonolysis, would split the Rh-MOP into two equal fragments. We hypothesize that this splitting would release the metal-organic macrocycle built up from the three 4-c Rh(II) paddle-wheel units linked to each other by the three $\mathrm{L}_{3}$ linkers and with six pendant partly deprotonated isophthalic linkers.

We synthesized isoreticular Rh-MOP by heating a $N, N$-dimethylacetamide (DMA) suspension of $\mathrm{L}_{3}, \mathrm{~L}_{5}, \mathrm{Na}_{2} \mathrm{CO}_{3}$ and $\mathrm{Rh}_{2}(\mathrm{OAc})_{4}$ at $100{ }^{\circ} \mathrm{C}$. After 4 days, the reaction yielded a dark precipitate that was centrifuged to separate out the $\mathrm{Na}_{2} \mathrm{CO}_{3}$. The resultant solution was precipitated in $\mathrm{MeOH}$ to yield a green powder, which was washed several times with $\mathrm{MeOH}$. Finally, diffusion of diethyl ether into a DMF solution of this green powder afforded green parallelogram-shaped crystals of Rh-MOP. SCXRD confirmed the formation of the expected isoreticular MOP, built up from six $\mathrm{L}_{3}$ linkers and six cleavable $\mathrm{L}_{5}$ linkers (Fig. 3A). Next, the six linkers were cleaved to synthesize the metal-organic macrocycle by bubbling ozone through a suspension of Rh-MOP in DMA and hydrogen peroxide $\left(50\right.$ wt. \% in $\mathrm{H}_{2} \mathrm{O}$ ) at room temperature for $30 \mathrm{~min}$. Note that oxidative conditions were used in this clip-off reaction to 
obtain a pure Rh-macrocycle functionalized with six carboxylic acid groups. Afterwards, the resulting green suspension was centrifuged, and an aqueous $\mathrm{HCl}$ solution was added to the supernatant to precipitate the Rh-macrocycle as a green solid. This solid was finally separated by centrifugation, washed with water three times, and dried at $85^{\circ} \mathrm{C}$ under vacuum.

We confirmed formation of the expected metal-organic Rh-macrocycle by ${ }^{1} \mathrm{H}$ NMR of the digested ozonated Rh-MOP (5\% HF/DMSO- $d_{6}$; Fig. 3B), MALDI-TOF mass (Fig. 3C) spectrometry and UV-Vis spectroscopy (fig. S33). The ${ }^{1} \mathrm{H}$ NMR spectrum lacked all the characteristic peaks of $\mathrm{L}_{5}$ (olefinic protons at $\delta=7.43 \mathrm{ppm}$; and phenyl protons at $\delta=8.18$ ppm, $7.92 \mathrm{ppm}, 7.85 \mathrm{ppm}$ and $7.51 \mathrm{ppm}$ ), yet it did reveal the characteristic signals of $\mathrm{L}_{3}$ (phenyl protons at $\delta=8.30 \mathrm{ppm}$ and $8.16 \mathrm{ppm}$; and tert-butyl protons at $\delta=1.33 \mathrm{ppm}$ ) and those of isophthalic acid (phenyl protons at $\delta=8.47 \mathrm{ppm}, 8.17 \mathrm{ppm}$ and $7.65 \mathrm{ppm}$; Fig. 3B). This integrated spectrum indicated an $\mathrm{L}_{3}$ /isophthalic acid proton ratio of 1:2, consistent with the formula expected for the Rh-macrocycle (figs. S31,32). Moreover, the absence of any proton signal corresponding to 3-formylbenzoic acid corroborated full cleavage of all the olefinic bonds in the Rh-MOP and their subsequent transformation to carboxylic acid groups.

Finally, we unambiguously confirmed the structure of the targeted macrocycle by positive mode MALDI-TOF analysis of pristine and ozonated samples of the Rh-MOP. The MALDI-TOF spectrum of the Rh-MOP showed its molecular ion $[\mathrm{M}+\mathrm{H}]^{+}(\mathrm{m} / \mathrm{z}=4651.5)$. Contrariwise, in the spectrum of the ozonated Rh-MOP, this peak was absent, whereas a new peak, for the molecular ion $[\mathrm{M}+\mathrm{H}]^{+}(\mathrm{m} / \mathrm{z}=2267.7)$ corresponding to the Rh-macrocycle, was present (Fig. 3C). Moreover, UV-Vis analysis of both pristine and ozonated Rh-MOP samples did not reveal any shift in the low-energy absorption band I at $592 \mathrm{~nm}$, indicating that the coordination of $\mathrm{Rh}(\mathrm{II})$ ions in the ozonated solid had not changed, thus further corroborating formation of the Rh-complex (fig. S33). 
A
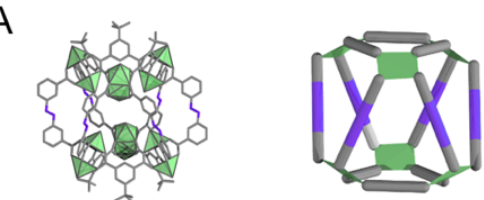

Rh-MOP- $\left(\mathrm{L}_{3}-\mathrm{L}_{4}\right)$

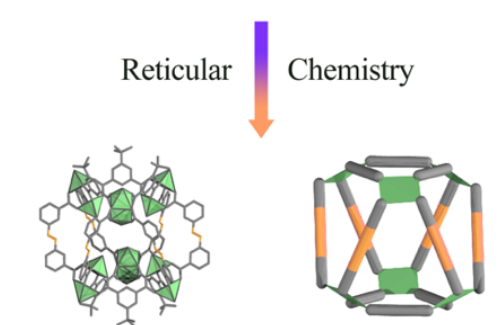

Rh-MOP- $\left(\mathrm{L}_{3}-\mathrm{L}_{5}\right)$
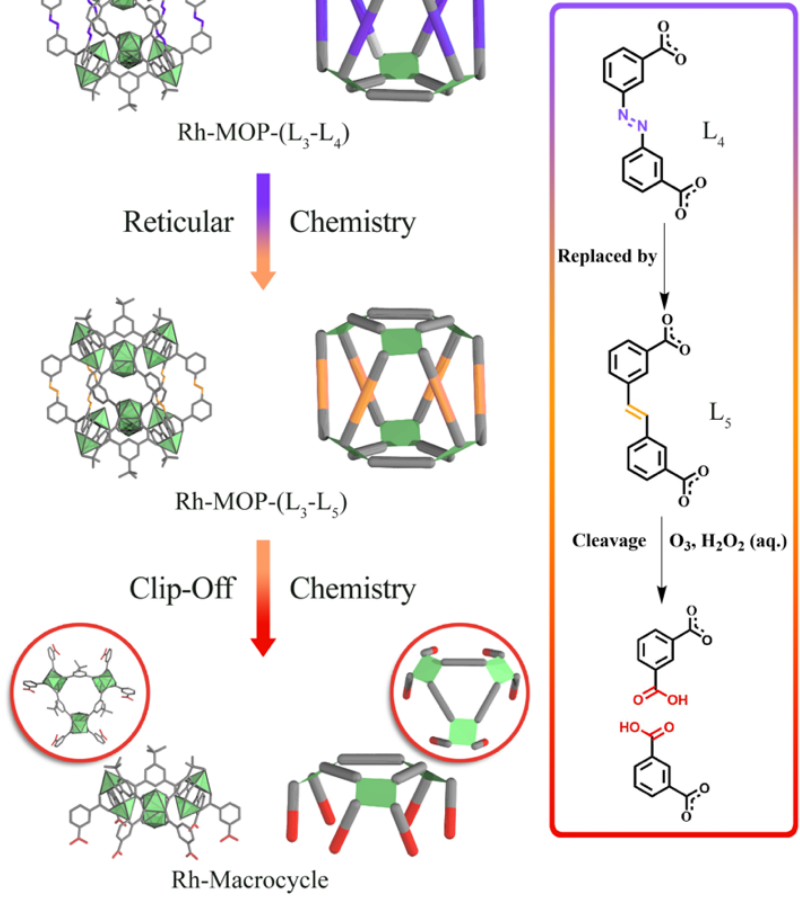

B
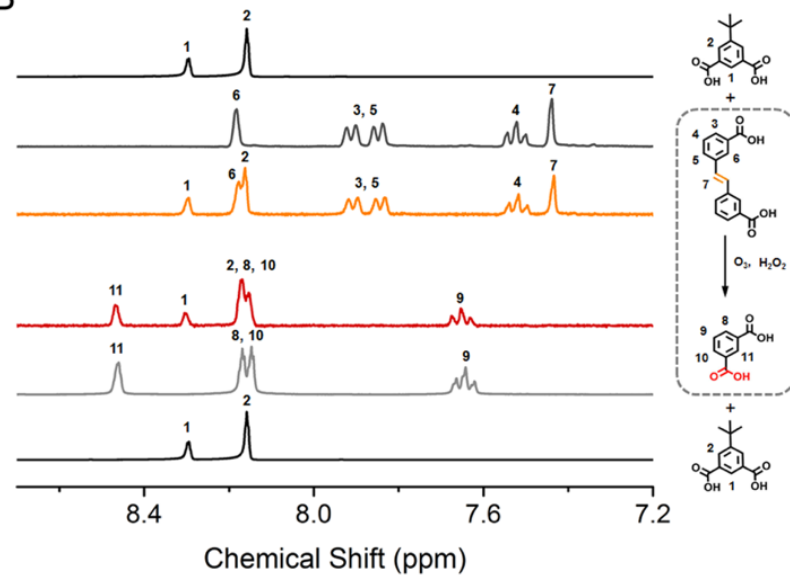

On, $\mathrm{H}_{2} \mathrm{a}$

C

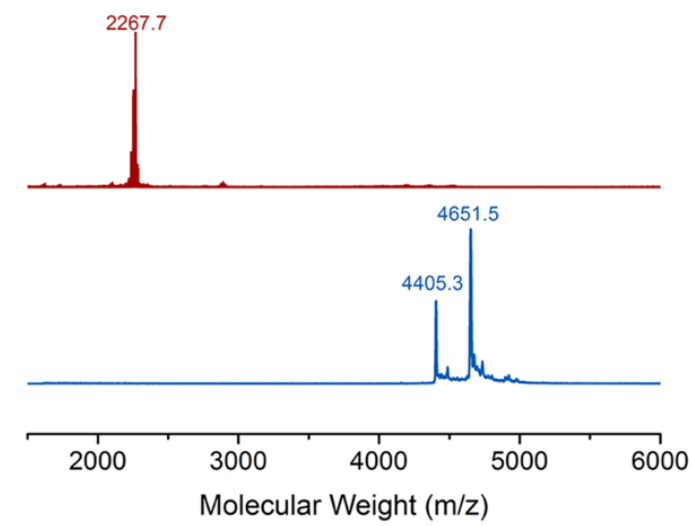

Fig 3. Clip-off synthesis of a triangular Rh-macrocycle. (A) Schematic representation of the synthesis of a Rh-macrocycle from a discrete mixed-linker Rh-MOP, in which a linker containing an olefinic bond had been incorporated by reticular chemistry previous to ozonolysis. (B) ${ }^{1} \mathrm{H}$ NMR spectra $\left(360 \mathrm{MHz}, \mathrm{DMSO}-d_{6}\right)$ of digested $\mathrm{L}_{3}$ (black), $\mathrm{L}_{5}$ (dark grey), 
Rh-MOP (orange), Rh-macrocycle (red), and isophthalic acid (light grey). Assigned proton signals for digested Rh-MOP (orange): $\delta_{1}=8.30(\mathrm{~s}, 1 \mathrm{H}), \delta_{2}=8.16(\mathrm{~s}, 2 \mathrm{H}), \delta_{3,5}=7.92(\mathrm{~d}, J=$ $7.7 \mathrm{~Hz}, 2 \mathrm{H}), \delta_{3,5}=7.85(\mathrm{~d}, J=7.7 \mathrm{~Hz}, 2 \mathrm{H}), \delta_{4}=7.51(\mathrm{t}, J=7.7 \mathrm{~Hz}, 2 \mathrm{H}), \delta_{6}=8.18(\mathrm{~s}, 2 \mathrm{H})$, and $\delta_{7}=7.43(\mathrm{~s}, 2 \mathrm{H})$. Assigned proton signals for digested Rh macrocycle (red): $\delta_{1}=8.30(\mathrm{~s}, 1 \mathrm{H})$, $\delta_{2,8,10}=8.17-8.15(\mathrm{~m}, 6 \mathrm{H}), \delta_{9}=7.65(\mathrm{t}, J=7.8 \mathrm{~Hz}, 2 \mathrm{H})$, and $\delta_{11}=8.47(\mathrm{~s}, 2 \mathrm{H})$. (C) MALDITOF spectrometry of Rh-MOP (blue) and Rh-macrocycle fully functionalized with $\mathrm{COOH}$ (red). The mass corresponding to the formula $\left[\mathrm{Rh}_{12}\left(\mathrm{~L}_{3}\right)_{6}\left(\mathrm{~L}_{5}\right)_{6}(\mathrm{DMA})\left(\mathrm{H}_{2} \mathrm{O}\right)_{2}(\mathrm{MeOH})_{4}+\mathrm{H}\right]^{+}$ $($ expected $=4403.8$; found $=4405.3)$ and $\left[\mathrm{Rh}_{12}\left(\mathrm{~L}_{3}\right)_{6}\left(\mathrm{~L}_{5}\right)_{6}\left(\mathrm{H}_{2} \mathrm{O}\right)_{2}(\mathrm{DMA})_{2}\left(\mathrm{CH}_{3} \mathrm{CN}\right)_{7}+\mathrm{H}\right]^{+}$ (expected $=4650.0$; found $=4651.5$ ) have been highlighted for the Rh-MOP sample. In the case of the Rh-macrocycle, the mass corresponding to the formula $\left[\mathrm{Rh}_{6}\left(\mathrm{~L}_{3}\right)_{3}\left(\mathrm{C}_{8} \mathrm{H}_{5} \mathrm{O}_{4}\right)_{6}+\mathrm{H}\right]^{+}$ has been highlighted $($ expected $=2268.8$; found $=2267.7$ ).

\section{Summary}

Herein we have presented Clip-off Chemistry, a new approach to synthesizing molecules and materials based on selective bond-breaking in reticular precursors materials via common organic transformations. In this approach, programmed de-reticulation and controlled etching occur at the molecular level. As proof-of-concept, we transformed two 3-D MOFs (Zr-scuMOF and Sc-soc-MOF) into two topologically distinct 3-D MOFs (Zr-pcu-MOF and Sc-pcuMOF, respectively). Moreover, we extended the Clip-off Chemistry to a 0-D system, by transforming a MOP into a metal-organic macrocycle. In the examples that we reported here, we demonstrated that the cleavage was quantitative and selective; was effective both in solidphase and solution-phase chemistry; and occurred in a single-crystal-to-single crystal fashion across the entire bulk precursor sample. Moreover, we showed control on the cleavage of each olefinic bond into carboxylic acid groups using oxidative conditions. Our preliminary results suggest that Clip-off Chemistry is ripe for further exploration. Since it is based on bond cleavage, the dimensionality of the parent reticular material dictates the synthetic feasibility of the target molecules and/or materials (Fig. 4). Thus, it offers the possibility to modify structures of reticular materials without changing their dimensionality (e.g. 3-D $\rightarrow 3-\mathrm{D}$, or 2-D $\rightarrow 2-\mathrm{D}$ ). For example, one might employ Clip-off Chemistry for post-synthetic modification of the topology and/or the porosity of a given material. Moreover, it could be applied to 3-D structures to augment the diversity of new 2-D materials: for instance, by converting well-known 3-D pillared reticular materials into isolated 2-D layers, via selective clipping of the pillaring linkers. Similarly, it shows promise for using 3-D or 2-D structures to isolate 1-D materials in the form of metallic chains, or to synthesize 0-D systems, including MOPs, macrocycles and clusters, from 3-D/2-D/1-D structures and even from other 0-D systems (e.g. MOPs). Cleavage of bonds in reticular materials may also be expanded to purely organic structures. Indeed, COFs and organic cages are another endless source of precursors in Clip-off Chemistry, from which, for example, organic polymers, cages, macrocycles or other organic compounds could be synthesized. Similarly, other reactions involving the cleavage of bonds (e.g. photolysis) are potential candidates to be applied in reticular materials for their programmable disassembly (39-41). In summary, the results presented herein prove that the programmed disassembly of reticular materials is feasible, and that it can become an innovative new synthetic tool that researchers will use to synthesize myriad molecular architectures. 


\section{D 2D $\quad$ 1D OD}

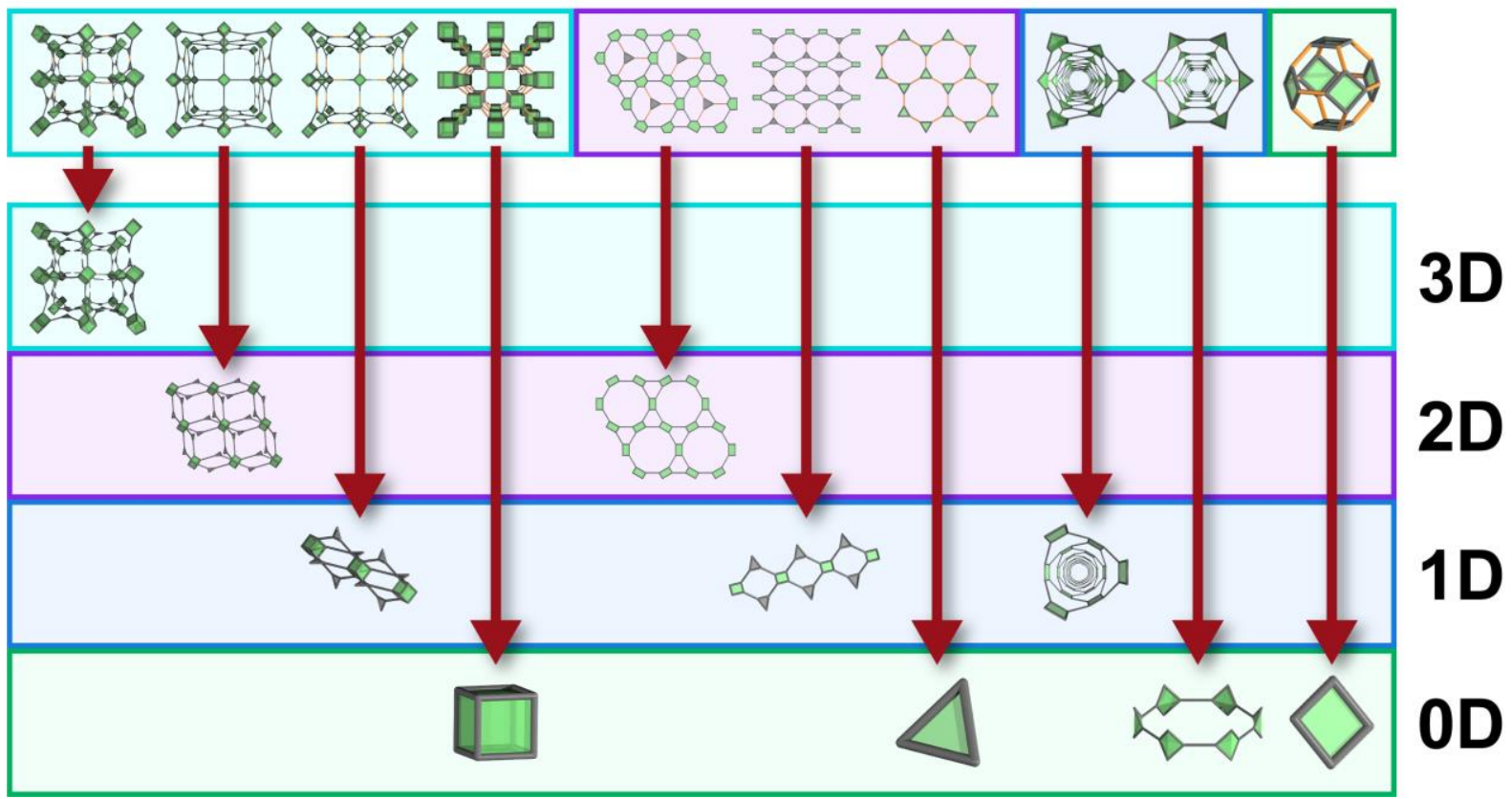

Fig 4. Clip-off Chemistry for the synthesis of molecular architectures of different dimensionality. Schematic illustrating the potential outcomes of clip-off chemistry, in which the dimensionality of the parent reticular material dictates the dimensionality of the target molecules and structures.

\section{REFERENCES AND NOTES:}

1. H. Li, M. Eddaoudi, T. L. Groy, O. M. Yaghi, Establishing microporosity in open metalorganic frameworks: gas sorption isotherms for $\mathrm{Zn}(\mathrm{BDC}) \quad(\mathrm{BDC}=1,4-$ benzenedicarboxylate). J. Am. Chem. Soc. 120, 8571-8572 (1998).

2. O. M. Yaghi, M. O'Keeffe, N. W. Ockwig, H. K. Chae, M. Eddaoudi, J. Kim, Reticular synthesis and the design of new materials. Nature 423, 705-714 (2003).

3. H. Li, M. Eddaoudi, M. O'Keeffe, O. M. Yaghi, Design and synthesis of an exceptionally stable and highly porous metal-organic framework. Nature 402, 276-279 (1999).

4. O. M. Yaghi, G. Li, H. Li, Selective binding and removal of guests in a microporous metalorganic framework. Nature 378, 703-706 (1995).

5. S. Kitagawa, R. Kitaura, S. Noro, Functional porous coordination polymers. Angew. Chem. Int. Ed. 43, 2334-2375 (2004).

6. B. F. Abrahams, B. F. Hoskins, D. M. Michail, R. Robson, Assembly of porphyrin building blocks into network structures with large channels. Nature 369, 727-729 (1994).

7. B. F. Hoskins, R. Robson, Infinite polymeric frameworks consisting of three dimensionally linked rod-like segments. J. Am. Chem. Soc. 111, 5962-5964 (1989).

8. O. M. Yaghi, M. J. Kalmutzki, C. S. Diercks, Introduction to Reticular Chemistry: MetalOrganic Frameworks and Covalent Organic Frameworks (Wiley, 2019).

9. X. Feng, X. Ding, D. Jiang, Covalent organic frameworks. Chem. Soc. Rev. 41, 6010-6022 (2012).

10. D. J. Tranchemontagne, Z. Ni, M. O'Keeffe, O. M. Yaghi, Reticular chemistry of metalorganic polyhedra. Angew. Chem. Int. Ed. 47, 5136-5147 (2008). 
11. J. J. Perry IV, J. A. Perman, M. J. Zaworotko, Design and synthesis of metal-organic frameworks using metal-organic polyhedra as supermolecular building blocks. Chem. Soc. Rev. 38, 1400-1417 (2009).

12. S. Lee, H. Jeong, D. Nam, M. S. Lah, W. Choe, The rise of metal-organic polyhedra. Chem. Soc. Rev. 50, 528-555 (2021).

13. M. Eddaoudi, D. B. Moler, H. Li., B. Chen, T. M. Reineke, M. O'Keeffe, O. M. Yaghi, Modular chemistry: secondary building units as a basis for the design of highly porous and robust metal-organic carboxylate frameworks. Acc. Chem. Res. 34, 319-330 (2001).

14. M. Eddaoudi, J. Kim, N. Rosi, D. Vodak, J. Wachter, M. O'Keeffe, O. M. Yaghi, Systematic design of pore size and functionality in isoreticular MOFs and their application in methane storage. Science 295, 469-472 (2002).

15. S. M. Cohen, Postsynthetic methods for the functionalization of metal-organic frameworks. Chem. Rev. 112, 970-1000 (2012).

16. O. M. Yaghi, Reticular chemistry: molecular precision in infinite 2D and 3D. Mol. Front. J. 3, 66-83 (2019).

17. M. O'Keeffe, O. M. Yaghi, Deconstructing the crystal structures of metal-organic frameworks and related materials into their underlying nets. Chem. Rev. 112, 675-702 (2012).

18. M. O'Keeffe, M. A. Peskov, S. J. Ramsden, O. M. Yaghi, The reticular chemistry structure resource (RCSR) database of, and symbols for, crystal nets. Acc. Chem. Res. 41, 1782-1789 (2008).

19. V. A. Blatov, A. P. Shevchenko, D. M. Proserpio, Applied topological analysis of crystal structures with the program package Topospro. Cryst. Growth Des. 14, 3576-3586 (2014).

20. A. F. Wells, Three-Dimensional Nets and Polyhedral (Wiley, 1977).

21. H. Wang, X. Dong, J. Lin, S. J. Teat, S. Jensen, J. Cure, E. V. Alexandrov, Q. Xia, K. Tan, Q. Wang, D. H. Olson, D. M. Proserpio, Y. J. Chabal, T. Thonhauser, J. Sun, Y. Han, J. Li, Topologically guided tuning of Zr-MOF pore structures for highly selective separation of $\mathrm{C}_{6}$ alkane isomers. Nat. Commun. 9, 1745 (2018).

22. M. Li, D. Li, M. O'Keeffe, O. M. Yaghi, Topological analysis of metal-organic frameworks with polytopic linkers and/or multiple building units and the minimal transitivity principle. Chem. Rev. 114, 1343-1370 (2014).

23. J. Albalad, H. Xu, F. Gándara, M. Haouas, C. Martineau-Corcos, R. Mas-Ballesté, S. A. Barnett, J. Juanhuix, I. Imaz, D. Maspoch, Single-crystal-to-single-crystal postsynthetic modification of a metal-organic framework via ozonolysis. J. Am. Chem. Soc. 140, 20282031 (2018).

24. V. Guillerm, H. Xu, J. Albalad, I. Imaz, D. Maspoch, Postsynthetic selective ligand cleavage by solid-gas phase ozonolysis fuses micropores into mesopores in metal-organic frameworks. J. Am. Chem. Soc. 140, 15022-15030 (2018).

25. R. Criegee, Mechanism of ozonolysis. Angew. Chem. Int. Ed. 14, 745-752 (1975).

26. C. E. Schiaffo, P. H. Dussault, Ozonolysis in solvent/water mixtures: direct conversion of alkenes to aldehydes and ketones. J. Org. Chem. 73, 4688-4690 (2008).

27. L. B. Harding, A. W. Goddard, Mechanisms of gas-phase and liquid-phase ozonolysis. $J$. Am. Chem. Soc. 100, 7180-7188 (1978).

28. L. H. Xie, X. M. Liu, T. He, J. R. Li, Metal-organic frameworks for the capture of trace aromatic volatile organic compounds. Chem 4, 1911-1927 (2018).

29. S. Wang, L. Chen, M. Wahiduzzaman, A. Tissot, L. Zhou, I. A. Ibarra, A. GutiérrezAlejandre, J. S. Lee, J. S. Chang, Z. Liu, J. Marrot, W. Shepard, G. Maurin, Q. Xu, C. Serre, A mesoporous zirconium-isophthalate multifunctional platform. Matter 4, 182-194 (2021). 
30. H. Furukawa, F. Gándara, Y. B. Zhang, J. Jiang, W. L. Queen, M. R. Hudson, O. M. Yaghi, Water adsorption in porous metal-organic frameworks and related materials. J. Am. Chem. Soc. 136, 4369-4381 (2014).

31. J. W. Zhang, P. Qu, M. C. Hu, S. N. Li, Y. C. Jiang, Q. G. Zhai, Topology-guided design for Sc-soc-MOFs and their enhanced storage and separation for $\mathrm{CO}_{2}$ and $\mathrm{C}_{2}$-hydrocarbons. Inorg. Chem. 58, 16792-16799 (2019).

32. Y. Liu, J. F. Eubank, A. J. Cairns, J. Eckert, V. C. Kravtsov, R. Luebke, M. Eddaoudi, Assembly of metal-organic frameworks (MOFs) based on indium-trimer building blocks: a porous MOF with soc topology and high hydrogen storage. Angew. Chem. Int. Ed. 46, 3278-3283 (2007).

33. P. J. Stang, B. Olenyuk, Self-assembly, symmetry and molecular architecture: coordination as the motif in the rational design of supramolecular metallacyclic polygons and polyhedra. Acc. Chem. Res. 30, 502-518 (1997).

34. B. Olenyuk, A. Fechtenkötter, P. J. Stang, Molecular architecture of cyclic nanostructures: use of co-ordination chemistry in the building of supermolecules with predefined geometric shapes. J. Chem. Soc., Dalton Trans. 1998, 1707-1728 (1998).

35. M. Fujita, O. Sasaki, T. Mitsuhashi, T. Fujita, J. Yazaki, K. Yamaguchi, K. Ogura, On the structure of transition-metal-linked molecular squares. Chem. Commun. 1996, 1535-1536 (1996).

36. F. A. Cotton, L. M. Daniels, C. Lin, C. A. Murillo, Square and triangular arrays based on $\mathrm{Mo}_{2}{ }^{4+}$ and $\mathrm{Rh}_{2}{ }^{4+}$ units. J. Am. Chem. Soc. 121, 4538-4539 (1999).

37. Unpublished results.

38. J. R. Li, H. C. Zhou, Bridging-ligand-substitution strategy for the preparation of metalorganic polyhedra. Nat. Chem. 2, 893-898 (2010).

39. L. Feng, K. Y. Wang, G. S. Day, M. R. Ryder, H. C. Zhou, Destruction of metal-organic frameworks: positive and negative aspects of stability and lability. Chem. Rev. 120, 1308713133 (2020).

40. K. Y. Wang, L. Feng, T. H. Yan, S. Wu, E. A. Joseph, H. C. Zhou, Rapid generation of hierarchically porous metal-organic frameworks through laser photolysis. Angew. Chem. Int. Ed. 59, 11349-11354 (2020).

41. P. Shieh, Hill, M. R. Hill, W. Zhang, S. L. Kristufek, J. A. Johnson, Clip chemistry: diverse (bio)(macro) molecular and material function through breaking covalent bonds. Chem. Rev. 2021, 0c01282 (2021).

\section{ACKNOWLEDGMENTS}

We thank Thais Grancha, Jordi Martínez-Esaín and Teodor Parella for technical assistance. Funding: This work was supported by the Spanish MINECO (projects RTI2018-095622-BI00 and PID2019-106403RB-I00) and the Catalan AGAUR (project 2017 SGR 238). It was also funded by the CERCA Programme /Generalitat de Catalunya. ICN2 is supported by the Severo Ochoa programme from the Spanish MINECO (Grant No. SEV-2017-0706). F.G. acknowledges funding from the Spanish Research Agency (AEI, CTQ2017-87262-R, EUR2019-103824). The project that generated these results received support from a fellowship (LCF/BQ/PR20/11770011) of the "la Caixa" Foundation (ID 100010434). Y.Y. acknowledges the China Scholarship Council for scholarship support. Author contributions: Y.Y., B.O.R. and V.G. performed the design and synthesis of the 3-D MOFs via clip-off chemistry. A.B.R. and A.C.S. performed the design and synthesis of the Rh-macrocycle via clip-off chemistry. F.G., J.J. and I.I. performed SCXRD experiments and resolution. S.J. and F.B. contributed on the synthesis of non-commercial linkers and advised on the project. D.M. wrote the manuscript with substantial input from all authors. I.I. and D.M. participated in conceptual development. 
Competing interests: the authors declare no competing interests. Data and materials availability: All data are available in the main text or in the supplementary materials. 